\title{
The heterogeneity of lung macrophages in the susceptibility to disease
}

\author{
Luisa Morales-Nebreda ${ }^{1}$, Alexander V. Misharin ${ }^{2}$, Harris Perlman ${ }^{2}$ and \\ G.R. Scott Budinger ${ }^{1}$
}

Affiliations: ${ }^{1}$ Dept of Medicine, Division of Pulmonary and Critical Care Medicine, Northwestern University, Feinberg School of Medicine, Chicago, IL, USA. ${ }^{2}$ Dept of Medicine, Division of Rheumatology, Northwestern University, Feinberg School of Medicine, Chicago, IL, USA.

Correspondence: G.R. Scott Budinger, Northwestern University, Feinberg School of Medicine, Dept of Medicine, Division of Pulmonary and Critical Care Medicine, 240 East Huron Street, Room M-343, Chicago, IL 60611, USA. E-mail: s-budinganorthwestern.edu

ABSTRACT Alveolar macrophages are specialised resident phagocytes in the alveolus, constituting the first line of immune cellular defence in the lung. As the lung microenvironment is challenged and remodelled by inhaled pathogens and air particles, so is the alveolar macrophage pool altered by signals that maintain and/or replace its composition. The signals that induce the recruitment of circulating monocytes to the injured lung, as well as their distinct gene expression profile and susceptibility to epigenetic reprogramming by the local environment remain unclear. In this review, we summarise the unique characteristics of the alveolar macrophage pool ontogeny, phenotypic heterogeneity and plasticity during homeostasis, tissue injury and normal ageing. We also discuss new evidence arising from recent studies where investigators described how the epigenetic landscape drives the specific gene expression profile of alveolar macrophages. Altogether, new analysis of macrophages by means of "omic" technologies will allow us to identify key pathways by which these cells contribute to the development and resolution of lung disease in both mice and humans.

@ERSpublications

Alveolar macrophages: the influence of ontogeny and microenvironment on epigenetically programmed responses to stress http://ow.ly/Oweil

\section{Alveolar macrophage heterogeneity}

Alveolar macrophages are tissue-resident cells that line the inner epithelial surface of the alveoli and play a crucial role in lung development, surfactant homeostasis and immune surveillance [1]. By interacting with epithelial, stromal and immune cells in the lung, they maintain alveolar integrity and orchestrate responses to environmental challenges. Investigators have long suggested that alveolar macrophages originate from circulating blood monocytes. This concept was based on the observations that bone marrow-derived monocytes can repopulate the alveolar macrophage pool after lethal irradiation and are recruited to the lung in response to injury [1-4]. Recently, several investigative teams have challenged this paradigm. Using advanced lineage-tracing techniques in mice, they demonstrated that alveolar macrophages, which are absent at birth, originate from fetal monocytes, which populate the alveolar space in the first days of postnatal life. These fetal monocyte-derived alveolar macrophages are long-living cells, which are maintained via self-renewal, without replenishment from circulating bone marrow-derived monocytes (fig. 1) [5-7]. At the same time, other groups have used new techniques (RNA sequencing and chromatin immunoprecipitation (ChIP) sequencing) to compare the transcriptome and epigenome of resident macrophage populations in different tissues, revealing marked tissue-specific heterogeneity in their epigenetic landscape and resulting gene expression profile, which are driven by signals originating from the tissue microenvironment [8-10].

Received: April 62015 | Accepted after revision: May 52015

Conflict of interest: None declared.

Provenance: Submitted article, peer reviewed.

Copyright OERS 2015. ERR articles are open access and distributed under the terms of the Creative Commons Attribution Non-Commercial Licence 4.0. 


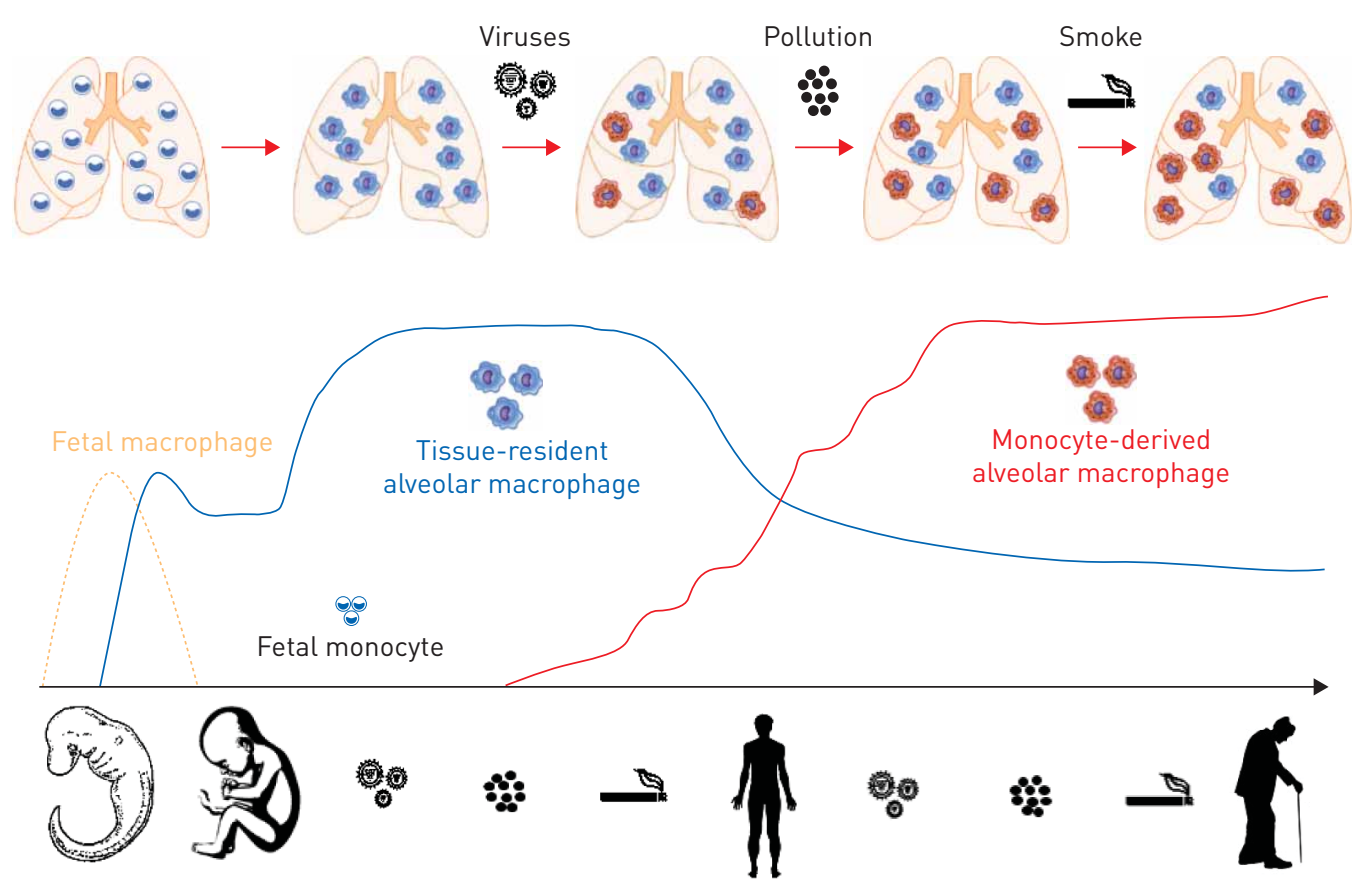

FIGURE 1 Lung macrophage development and composition through the lifespan. In the mouse, fetal monocyte cells that populate the lung during early embryogenesis differentiate into alveolar macrophages shortly after birth. This population of "tissue-resident" alveolar macrophages are long-lived and capable of self-renewal in homeostatic conditions (blue line). However, exposure to environmental challenges including viruses, air pollution and cigarette smoke over the lifespan may induce the recruitment of monocytes that differentiate into alveolar macrophages in response to cues provided by the local tissue microenvironment in the lung (red line). Over time, these monocyte-derived alveolar macrophages might persist in the lung and/or replace the tissue-resident alveolar macrophages.

These new data raise several important questions that challenge our consideration of alveolar macrophages as a homogenous cell population over the lifespan: do circulating monocytes that differentiate into alveolar macrophages during injury persist in the lung after resolution of the injury, and, if they do, is the epigenetic programming of these monocyte-derived alveolar macrophages the same as tissue-resident alveolar macrophages? Does macrophage ontogeny (tissue-resident compared with monocyte-derived) play a role in the response of alveolar macrophages to environmental challenges? Does the replacement of tissue-resident with monocyte-derived macrophages over the lifespan play a role in the age-related susceptibility to some lung diseases? Does damage to the lung parenchyma affect the tissue microenvironment in a way that interferes with the epigenetic reprogramming of recruited monocytes and, if so, does this alter the immune response to environmental challenges? While the answers to these questions are not yet known, recent advances in murine genetics, flow cytometry, single cell "omic" technologies and bioinformatics offer immediate promise. In addition, many of these technologies can now be applied to human tissues, allowing findings in murine studies to serve as a template to identify factors expressed or secreted by alveolar macrophages that might drive or ameliorate lung disease.

\section{Lung macrophage pool: ontogeny, composition and renewal capacity}

Alveolar macrophages form $90-95 \%$ of the cellular content within the alveoli under normal conditions, making them the natural "gatekeepers" of the respiratory system [1,4]. Alveolar macrophages reciprocally interact with alveolar epithelial cells, dendritic cells and T-cells via a network of cell surface receptors and chemokines/cytokines to finely tune the immune response to environmental pathogens and particulates in the lung $[1,11]$. Because they express proteins known to dampen the immune response to injury and facilitate the noninflammatory removal of apoptotic cells and debris, many investigators suggest that the role of the alveolar macrophage is to clear pathogens and particulates without inducing an inflammatory response that might disrupt gas exchange. Examples of anti-inflammatory molecules highly expressed in alveolar macrophages include the CD200 receptor, which binds its ligand expressed on alveolar epithelial cells to inhibit Toll-like receptor-induced inflammatory responses [12]. In addition, signal-regulatory protein- $\alpha$, expressed on alveolar macrophages, binds surfactant proteins A and D to inhibit macrophage activation and phagocytosis [1, 13]. Alveolar macrophages also release transforming growth factor (TGF)- $\beta$, which inhibits immune responses by preventing dendritic cell-mediated activation of effector T-cells $[4,14,15]$. In addition, alveolar macrophages express several scavenger receptors that bind 
complement, damage-associated molecular pattern molecules, pathogen-associated molecular pattern molecules and immunoglobulins to facilitate pathogen clearance $[2,16]$.

When injury is sufficiently severe, circulating monocytes are recruited to the lung where they differentiate into alveolar macrophages. The signals that induce the recruitment of circulating monocytes to the injured lung remain unclear; however, some data suggest that tissue-resident alveolar macrophages tend to inhibit this response [17]. As they differentiate, recruited monocytes express a host of pro-inflammatory and pro-fibrotic genes and can upregulate major histocompatibility complex class II, rendering them capable of activating effector T-cells through antigen presentation [18, 19]. Both tissue-resident and monocyte-derived alveolar macrophages express CD64 (Fc receptor that binds immunoglobulin G-type antibodies) and MER receptor tyrosine kinase bolstering efferocytosis [19]. Investigators have used mice deficient in CCR2, the receptor for monocyte chemoattractant protein-1, to investigate the specific role of monocyte-derived and tissue-resident macrophages in the response to inflammation [5, 6, 20-22]. Unfortunately, CCR2-deficient mice have monocytopenia, which synergises with the loss of CCR2 to limit the recruitment of monocytes into the lung and therefore provides little insight into the specific function or fate of monocyte-derived alveolar macrophages in disease.

Using parabiosis techniques and the generation of bone marrow chimeras with thoracic shielding in mice, investigators have reported that in the absence of lung injury, the tissue-resident alveolar macrophage pool persists for as long as 5-8 months, with minimal input from circulating monocytes [6, 23]. Tissue-resident alveolar macrophages may even survive mild influenza A infection or lipopolysaccharide (LPS)-induced lung injury, or proliferate to restore their population after injury $[6,23]$. Monocyte-derived alveolar macrophages persist in the lung for up to 14 days after influenza A infection and for as long as 38 days after bleomycin-induced injury; however, longer-term data are lacking [23, 24]. As currently available thoracic shielding techniques probably result in incomplete chimerism due to protection of the thoracic and sternal bone marrow, further refinement of lineage tracing techniques is required to allow separation of the alveolar macrophage populations over time and after the resolution of injury. These populations can be examined to determine whether the epigenetic programming that drives the differentiation of monocyte-derived alveolar macrophages is complete or whether monocyte-derived and tissue-resident alveolar macrophages retain epigenetic marks reflecting their ontogeny. If the latter is true, these two macrophage populations might differ in their ability to maintain a normal alveolar environment during steady-state conditions or may respond differently to subsequent insults.

\section{Macrophage plasticity in homeostasis and injury}

State-of-the-art technologies, such as flow cytometry and gene expression profiling provide the opportunity for detailed molecular phenotyping of immune cell populations in the normal and diseased lung. Our group used data from the Immunological Genome (ImmGen) Consortium [10] to develop a panel of antibodies that can identify all myeloid cell populations in standardised lung tissue homogenates from the normal and diseased murine lung using multicolour flow cytometry [24]. Because this panel recognises surface markers, it can be used for sorting live cells for subsequent gene expression, epigenetic or other analysis. Specifically, this panel identifies alveolar macrophages, interstitial macrophages, Ly6C ${ }^{\text {hi }}$ classical monocytes, and Ly6C ${ }^{\text {low }}$ non-classical monocytes. In humans, standardised protocols for the isolation of alveolar macrophages are lacking and are largely based on cells obtained during bronchoalveolar lavage. In mice, we and others found that samples obtained from bronchoalveolar lavage fluid poorly represented the heterogeneity of pulmonary macrophages observed in lung homogenates [25, 26].

In carefully controlled cell culture conditions, the administration of bacterial LPS and interferon- $\gamma$ to murine macrophages results in a characteristic pattern of gene expression that includes increased expression of the pro-inflammatory genes tumour necrosis factor- $\alpha$, interleukin (IL)- 6 and inducible nitric oxide synthase, while the administration of IL- 4 results in increased expression of genes involved in tissue remodelling including IL-13, TGF- $\beta$ and arginase-1. This polarisation of macrophages into "M1" and "M2" gene expression phenotypes has proved useful for understanding the complexity of tissue macrophage function over the time course of tissue injury and repair [27, 28]. However, recent findings from several independent groups suggest that this concept incompletely describes the complexity of macrophage gene expression in vivo, which might include simultaneous expression of M1 and M2 genes during injury and repair [29]. Furthermore, the applicability of this paradigm to human lung macrophages is not known. These findings have led investigators to examine macrophage plasticity and function in vivo using unbiased techniques [29-31]. Advances in transcriptomic and epigenomic approaches, in particular RNA sequencing and ChIP sequencing allow for the analysis of the transcriptome in small cell populations and even in single cells [32]. Similar unbiased examination of the proteome in isolated immune cell populations is now technically feasible, but current technology requires more material than is available from a single mouse lung or from a small tissue biopsy specimen. Nevertheless, we can now 
envision the identification of key pathways by which macrophages contribute to the development and resolution of lung disease through analysis of the macrophage transcriptome, epigenome and proteome in health and disease in both mice and humans.

Irrespective of their ontogeny, alveolar macrophages demonstrate a pattern of gene expression distinct from macrophages in other tissues and from other myeloid cells [8-10]. Understanding the molecular basis for these differences represents an area of intense investigation. Recently, investigators have used different approaches to suggest that changes in histone modifications, i.e. the epigenetic landscape, drive the expression of alveolar macrophage-specific genes $[8,9]$. Specifically, these investigators have shown that enhancer regions distinguish alveolar macrophages from other myeloid cells and from other tissue-resident macrophage populations. These enhancer regions are defined as regions distal to the transcriptional start site of alveolar macrophage-specific genes that undergo specific histone modifications, including high levels of $\mathrm{H} 3 \mathrm{~K} 4 \mathrm{mel}$ and low levels of $\mathrm{H} 3 \mathrm{~K} 4 \mathrm{me} 3$, among others. These epigenetic modifications result in the recruitment of PU.1, which serves as a "pioneer" transcription factor, followed by recruitment of a host of transcription factors to the enhancer regions that augment alveolar macrophage-specific gene expression $[8,9]$. Furthermore, the adoptive transfer of peritoneal macrophages into the lung resulted in the expression of some alveolar macrophage-specific genes, suggesting that macrophage differentiation is at least partially reversible [9].

These results suggest that the lung microenvironment supplies continuous signals to maintain or replace the alveolar macrophage pool. While our understanding of these signals is incomplete, granulocyte macrophage colony-stimulating factor (GM-CSF) is known to play an important role. Humans and mice genetically deficient in GM-CSF or its receptor fail to develop alveolar macrophages capable of clearing surfactant protein from the lung, resulting in pulmonary alveolar proteinosis [5, 33, 34]. Pleotropic peroxisome proliferator-activated receptor- $\gamma$, important for lipid metabolism, may act downstream of GM-CSF to drive alveolar macrophage differentiation [35-37]. Another transcription factor, the B lymphoid transcription repressor BTB and CNC homology (Bach)2 has been linked with the development of pulmonary alveolar proteinosis independently of GM-CSF signalling, and Bach2-deficient animals showed increased expression of M2 macrophage phenotype-related genes, such as arginase-1 and chitinase-like 3 [38]. In addition, the host microbiota plays an important role in the modulation of the local immune response during the steady-state and diseased lung. Investigators have reported that changes in the microbiota during influenza-induced lung injury altered the gene expression profiling of alveolar macrophages rendering a decreased antiviral immune response to the infection [39]. Another group of investigators showed that priming the immune system with bacteria prior to influenza infection induced an M2 macrophage phenotype, which dampened lung injury [40]. It is tempting to speculate that the change in the lung microenvironment might alter the epigenetic programming of tissue-resident or monocyte-derived alveolar macrophages, which might change their response to subsequent environmental challenges.

\section{Conclusions}

Alveolar macrophages are critical resident cells in the alveolus important for both lung homeostasis and the response to injury. In the adult lung, there are at least two ontologically distinct populations of alveolar macrophages. Tissue-resident alveolar macrophages develop outside of the bone marrow, differentiate into alveolar macrophages shortly after birth, are capable of self-renewal, and persist over the lifespan. Monocyte-derived alveolar macrophages develop from circulating monocytes and are recruited to the lung during injury. While the molecular basis for the differentiation of monocytes into alveolar macrophages is incompletely understood, we now know that factors present in the lung microenvironment induce at least partially reversible changes in the epigenetic landscape of the cell, with the appearance of enhancer sites around differentially expressed alveolar macrophage genes. These findings suggest that alveolar macrophages might change their gene expression profiles during normal conditions or in response to environmental challenges as a result of age or injury-related replacement of tissue-resident with monocyte-derived alveolar macrophages, or as the result of changes in the lung microenvironment that impair the maintenance of the alveolar macrophage pool. Advances in flow cytometry and "omic" technologies in mice and humans will allow us to explore changes in macrophage populations over time and with disease with unprecedented detail, offering the hope for developing novel biomarkers and therapeutics.

\section{References}

Hussell T, Bell TJ. Alveolar macrophages: plasticity in a tissue-specific context. Nat Rev Immunol 2014; 14: 81-93.

2 Schneberger D, Aharonson-Raz K, Singh B. Monocyte and macrophage heterogeneity and Toll-like receptors in the lung. Cell Tissue Res 2011; 343: 97-106.

3 Scott CL, Henri S, Guilliams M. Mononuclear phagocytes of the intestine, the skin, and the lung. Immunol Rev 2014; 262: 9-24.

4 Kopf M, Schneider C, Nobs SP. The development and function of lung-resident macrophages and dendritic cells. Nat Immunol 2015; 16: 36-44. 
5 Guilliams M, De Kleer I, Henri S, et al. Alveolar macrophages develop from fetal monocytes that differentiate into long-lived cells in the first week of life via GM-CSF. J Exp Med 2013; 210: 1977-1992.

6 Hashimoto D, Chow A, Noizat C, et al. Tissue-resident macrophages self-maintain locally throughout adult life with minimal contribution from circulating monocytes. Immunity 2013; 38: 792-804.

7 Yona S, Kim KW, Worf Y, et al. Fate mapping reveals origins and dynamics of monocytes and tissue macrophages under homeostasis. Immunity 2013; 38: 79-91.

8 Gosselin D, Link VM, Romaoski CE, et al. Environment drives selection and function of enhancers controlling tissue-specific macrophage identities. Cell 2014; 159: 1327-1340.

9 Lavin Y, Winter D, Blecher-Gonen R, et al. Tissue-resident macrophage enhancer landscapes are shaped by the local microenvironment. Cell 2014; 159: 1312-1326.

10 Gautier EL, Shay T, Miller J, et al. Gene-expression profiles and transcriptional regulatory pathways that underlie the identity and diversity of mouse tissue macrophages. Nat Immunol 2012; 13: 1118-1128.

11 Westphalen K, Gusarova GA, Islam MN, et al. Sessile alveolar macrophages communicate with alveolar epithelium to modulate immunity. Nature 2014; 506: 503-506.

12 Snelgrove RJ, Goulding J, Didierlaurent AM, et al. A critical function for CD200 in lung immune homeostasis and the severity of influenza infection. Nat Immunol 2008; 9: 1074-1083.

13 Janssen WJ, McPhillips KA, Dickinson MG, et al. Surfactant proteins A and D suppress alveolar macrophage phagocytosis via interaction with SIRP $\alpha$. Am J Respir Crit Care Med 2008; 178: 158-167.

14 Huang X, Wu J, Zhu W, et al. Expression of the human integrin $\beta 6$ subunit in alveolar type II cells and bronchiolar epithelial cells reverses lung inflammation in $\beta 6$ knockout mice. Am J Respir Cell Mol Biol 1998; 19: 636-642.

15 Huang XZ, Wu JF, Cass D, et al. Inactivation of the integrin $\beta 6$ subunit gene reveals a role of epithelial integrins in regulating inflammation in the lung and skin. J Cell Biol 1996; 133: 921-928.

16 Gordon SB, Read RC. Macrophage defences against respiratory tract infections: the immunology of childhood respiratory infections. Br Med Bull 2002; 61: 45-61.

17 Archambaud C, Salcedo SP, Lelouard H, et al. Contrasting roles of macrophages and dendritic cells in controlling initial pulmonary Brucella infection. Eur J Immunol 2010; 40: 3458-3471.

18 Cheong C, Matos I, Choi JH, et al. Microbial stimulation fully differentiates monocytes to DC-SIGN/CD209 dendritic cells for immune T cell areas. Cell 2010; 143: 416-429.

19 Plantinga M, Guilliams M, Vanheerswynghels $\mathrm{M}$, et al. Conventional and monocyte-derived $\mathrm{CD}_{11 \mathrm{~b}^{+}}$dendritic cells initiate and maintain $\mathrm{T}$ helper 2 cell-mediated immunity to house dust mite allergen. Immunity 2013; 38: 322-335.

20 Kurihara T, Warr G, Loy J, et al. Defects in macrophage recruitment and host defense in mice lacking the CCR2 chemokine receptor. J Exp Med 1997; 186: 1757-1762.

21 Boring L, Gosling J, Chensue SW, et al. Impaired monocyte migration and reduced type 1 (Th1) cytokine responses in C-C chemokine receptor 2 knockout mice. J Clin Invest 1997; 100: 2552-2561.

22 Maus UA, Wellmann S, Hampl C, et al. CCR2-positive monocytes recruited to inflamed lungs downregulate local CCL2 chemokine levels. 2005; 288: L350-L358.

23 Janssen WJ, Barthel L, Muldrow A, et al. Fas determines differential fates of resident and recruited macrophages during resolution of acute lung injury. Am J Respir Crit Care Med 2011; 184: 547-560.

24 Misharin AV, Morales-Nebreda L, Mutlu GM, et al. Flow cytometric analysis of macrophages and dendritic cell subsets in the mouse lung. Am J Respir Cell Mol Biol 2013; 49: 503-510.

25 Zaynagetdinov R, Sherrill TP, Kendall PL, et al. Identification of myeloid cell subsets in murine lungs using flow cytometry. Am J Respir Cell Mol Biol 2013; 49: 180-189.

26 Soroosh P, Doherty TA, Duan W, et al. Lung-resident tissue macrophages generate Foxp $3^{+}$regulatory T cells and promote airway tolerance. J Exp Med 2013; 210: 775-788.

27 Murray PJ, Allen JE, Biswas SK, et al. macrophage activation and polarization: nomenclature and experimental guidelines. Immunity 2014; 41: 14-20.

28 Sica A, Mantovani A. Macrophage plasticity and polarization: in vivo veritas. J Clin Invest 2012; 122: 787-795.

29 Xue J, Schmidt SV, Sander J, et al. Transcriptome-based network analysis reveals a spectrum model of human macrophage activation. Immunity 2014; 40: 274-288.

30 Saeed S, Quitin J, Kerstens $\mathrm{HH}$, et al. Epigenetic programming of monocyte-to-macrophage differentiation and trained innate immunity. Science 2014; 345: 1251086.

31 Tha AK, Huang SC, Sergushichev A, et al. Network integration of parallel metabolic and transcriptional data reveals metabolic modules that regulate macrophage polarization. Immunity 2015; 42: 419-430.

32 Treutlein B, Brownfield DG, Wu AR, et al. Reconstructing lineage hierarchies of the distal lung epithelium using single-cell RNA-seq. Nature 2014; 509: 371-375.

33 Shibata Y, Berclaz PY, Chroneos ZC, et al. GM-CSF regulates alveolar macrophage differentiation and innate immunity in the lung through PU.1. Immunity 2001; 15: 557-567.

34 Trapnell BC, Whitsett JA. GM-CSF regulates pulmonary surfactant homeostasis and alveolar macrophage-mediated innate host defense. Annu Rev Physiol 2002; 64: 775-802.

35 Chawla A. Control of macrophage activation and function by PPARs. Circ Res 2010; 106: 1559-1569.

36 Odegaard JI, Ricardo-Gonzalez RR, Goforth MH, et al. Macrophage-specific PPAR $\gamma$ controls alternative activation and improves insulin resistance. Nature 2007; 447: 1116-1120.

37 Schneider C, Nobs SP, Kurrer M, et al. Induction of the nuclear receptor PPAR- $\gamma$ by the cytokine GM-CSF is critical for the differentiation of fetal monocytes into alveolar macrophages. Nat Immunol 2014; 15: 1026-1037.

38 Nakamura A, Ebina-Shibuya R, Itoh-Nakadai A, et al. Transcription repressor Bach2 is required for pulmonary surfactant homeostasis and alveolar macrophage function. J Exp Med 2013; 210: 2191-2204.

39 Abt MC, Osborne LC, Moticelli LA, et al. Commensal bacteria calibrate the activation threshold of innate antiviral immunity. Immunity 2012; 37: 158-170.

40 Wang J, Li F, Sun R, et al. Bacterial colonization dampens influenza-mediated acute lung injury via induction of M2 alveolar macrophages. Nat Commun 2013; 4: 2106. 